\title{
ONLINE DATA COLLECTION AS ADAPTATION IN CONDUCTING QUANTITATIVE AND QUALITATIVE RESEARCH DURING THE COVID-19 PANDEMIC
}

\author{
Moises C. Torrentira, Jr. ${ }^{\mathrm{i}}$ \\ orcid.org/0000-0003-3812-0182 \\ EnP., PhD, College of Development Management, \\ University of Southeastern Philippines, \\ Philippines
}

\begin{abstract}
:
This study was conducted to identify online data collection methodologies as an adaptation in the conduct of quantitative and qualitative research amidst the COVID-19 pandemic. While data collection has been hampered by the restrictions posed by the quarantine measures of the local governments around the globe, researchers need to strategize and adapt remote tools in data collection. Analyzing from the responses of 25 highly engaged researchers who served as participants of this study through videoconferencing, it was found out that data collection for quantitative research may be conducted by using online development tools via personal or institutional subscriptions, using google forms as a free and conventional platform, and by using QR Code to generate the electronic survey questionnaire. On the other hand, the data collection for qualitative research may be conducted by using diaries and reflections of participants to replace the typical direct observation, using telephone or mobile phone to conduct an interview, and by using video-conferencing for key informant interview and focus group discussion. Considering the practicality and feasibility of these data collection methods, researchers cannot be constrained by the quarantine and health protocols in pursuing reliable, honest, and high-quality research outputs.
\end{abstract}

Keywords: online data collection, adaptation, quantitative research, qualitative research, COVID-19 pandemic

\section{Introduction}

The COVID-19 pandemic did not only disrupt the common systems of the society, economy, health, environment, education, and politics worldwide. It also dissuaded the customary practices of conducting research particularly on the gathering of necessary

\footnotetext{
i Correspondence: email moises.torrentira@usep.edu.ph
} 
data. The researches that are affected first are studies that involve bringing groups of people together in close proximity (APA, 2020). Before the pandemic, collecting data for research can be easily done through an actual survey, face-to-face interview, focus group discussion, and extensive literature review. But all these became unworkable and difficult with the onset of the pandemic primarily due to quarantine restrictions and other health protocols implemented by the local governments. Hence, COVID-19 pandemic has created a challenging environment in the conduct of research (Dodds and Hess, 2020). For example, clinical researchers are caught off-guard and are forced to make decisions on research visits in their ongoing clinical trials (Padala et al., 2020). Researchers around the globe paused their work to avoid unnecessary burden on respondents and participants, relieve themselves from safety and health risk exposures, and due to the absence of logistical support (Townsend et al., 2020). Aside from that, Sy et al (2020) noted that many traditional data collection methods are becoming unfeasible during physical distancing while academic and research staff are working from home often through a skeletal workforce which can hamper the progression of research projects.

There is no doubt that the spread of severe acute respiratory syndrome coronavirus 2 (SARS-CoV-2) has already taken on pandemic proportions (Remuzzi and Remuzzi, 2020) with worldwide 31,174,627 confirmed cases, 962,613 confirmed deaths, and at least 235 countries and territories affected as of 23, September 2020 (WHO, 2020). Virtually, no part of the globe will remain unaffected except in the most isolated locations (Le Blanc, 2020). The numbers indicate that COVID-19 is unstoppable at the moment and the only way to handle it is to prevent or lessen the spread of the coronavirus by implementing preventive and protective measures such as social distancing, wearing of protective gears, community quarantines, and lockdowns to name a few. Although containment measures, for example, in China have reduced new cases by more than $90 \%$, this reduction is not the case elsewhere thus making a global response to prepare health systems imperative (Remuzzi and Remuzzi, 2020).

With the need to continue the conduct of research even amid the COVID-19 threat, the data collection methodologies and processes in both quantitative and qualitative researches need to be modified or innovated. Given the restrictive measures implemented by local governments in their areas of jurisdiction, researchers should find opportunities to collaborate and adopt other innovative methodologies used by other professionals. Instead of looking at the negative implications of the COVID-19 pandemic, researchers need to explore and be creative in pursuing data collection while upholding academic integrity and scholarly research. Researchers are encouraged to consider new and untapped techniques and methods in data collection to continue the conduct of scholarly research. 


\section{Objectives of the Study}

This study aimed to outline online data collection as adaptation in the conduct of both qualitative and quantitative types of research given the situation of the COVID-19 pandemic.

\subsection{Methods and Materials}

This study made use of the online interview data generated from the experiences of 25 academicians and professionals involved in research during the time of the pandemic. The interview data were processed through thematic content analysis to produce the themes (Torrentira, 2019) that best describe the innovative data collection techniques amidst the restrictions and protocols during the COVID-19 pandemic.

\subsection{Results and Discussion}

Thematic analysis of the participants' responses revealed the practical and innovative data collection techniques amidst the COVID-19 pandemic. These are the following:

\section{Data Collection for Quantitative Research during the COVID-19 Pandemic}

Quantitative research makes use of empirical data expressed numerically to represent the perception or experience of the respondents. As such, quantitative data are gathered, accumulated, encoded, and statistically processed to analyze and estimate the interaction of variables through a deductive process. This interaction of variables may lead to the likelihood of the emergence of one phenomenon or outcome stated in a hypothesis. According to Goertzen (2017), quantitative research provides estimates for a large population, indicates the extensiveness of the attitudes held by the respondents, provides results which can be condensed by statistics, has high precision, definition, and standard, allows for statistical comparison between various groups, and measures the level of occurrence, actions, or trends. In short, quantitative research generally focuses on measuring social reality by viewing the world as a reality that can be objectively determined so rigid guides in the process of data collection and analysis are very important (Sukamolson, 2007).

The most common data collection technique in quantitative research is the conduct of the survey. A survey is a process by which the participants of the study personally respond to the structured questionnaire produced by the researcher. The responses are then retrieved, encoded, and analyzed to generate statistical data in the form of graphs or tables. Vaske (2019) elaborated that the data collected from surveys can result in hundreds of variables and thousands of respondents in a rigid process of data encoding in the database, running preliminary analysis to detect any missing data or potential outliers, conducting reliability and validity tests, and converting the data to generate indices of underlying concepts. 
But during the COVID-19 pandemic, social distancing and quarantine protocols are strictly implemented by local governments across the globe. This poses a big challenge in the data collection through a traditional survey that requires individual interaction with a large number of respondents or participants. This is strictly prohibited during the pandemic to prevent the possible spread of the coronavirus. Hence, in the middle of these restrictions, an online survey as a technique in data collection becomes inevitable. An online survey is a process of distributing the instrument or the questionnaire to the target respondents using the online platforms. While the personal distribution of surveys is desirable in terms of improving response rate, online distribution on the other hand is a very feasible adaptation given current restrictions (Sy et al., 2020). Thematic analysis generated the following methods as adaptations in the conduct of online survey:

\subsection{Using online development tools via personal or institutional subscriptions}

Online surveys can be made easy with the aid of development tools or software. In the United States and India, Qualtrics is widely used to generate demographically and politically represented target respondents (Boas et al., 2020). Microsoft Office packages can also include access to Microsoft Forms which can be used to develop online surveys or questionnaires. Other tools that have been used by researchers such as Amazon Mechanical Turk and Survey Monkey for market surveys (Bentley et al, 2017), Survey Gizmo (Abd Halim et al., 2018), Research Now SSI, and Opinion Access (Kimball, 2019) among others.

\subsection{Using Google forms as a free and conventional online platform}

The distribution of electronic survey questionnaires can also be done without a cost employing google forms through the services of email, messenger, Facebook, WhatsApp, and other social media accounts. In this way, the respondents shall access an online survey through a link included in posts from a Facebook page or an intended message sent through email, messenger or WhatsApp specifically set up for the research (Barnes et al, 2020). Social media is a powerful tool that can be used to build connections and share information (Glazier and Topping, 2012). Hence, amidst the many restrictions on mobility during the COVID-19 pandemic, data collection through this remote online platform can be feasible, safe, and very convenient.

\subsection{Using QR Code to generate the electronic survey questionnaire}

Data collection can also be performed by encapsulating the google forms link of the survey questionnaire in the QR code. A QR code is a type of pf matrix barcode which is admired for its fast readability and greater storage capacity compared to standard UPC barcodes (Pandya and Galiyawala, 2014). In this way, the link shall be embedded in the QR Code containing the electronic survey questionnaire. It can be sent in email or social media accounts. Once captured by the camera of an android or IOS mobile phone, the respondent can easily retrieve the questionnaire and conveniently respond to the 
questions or items on his or her mobile phone. Pandya and Galiyawala (2014) added that with the technology of mobile phones constantly emerging, especially in the area of mobile internet access, QR codes seem to be an adequate tool to quickly and efficiently converse URLs to users. Researchers should then take advantage of this little technology in navigating possible means of reaching the target respondents to generate the desired number of research participants.

\subsection{Data Collection for Qualitative Research during the COVID-19 Pandemic}

Qualitative research is useful for exploring new topics or understanding complex issues to explain the behavior and beliefs of people and for identifying the social or cultural norms of a group or society (Hennink, 2020). As such, this type of research is very particular in building rapport with the participants to create a comfortable atmosphere for participant disclosure because qualitative researchers study participant's knowledge and practices (Flick, 2018). Considering this nature of engagement, data collection for qualitative research requires a high level of participant-researcher relationship wherein the earlier opens up himself to a free-flowing conversation while the latter navigate himself to the new context or paradigm to process and create new knowledge. There is somehow a personal fusion of horizon (Ramsbotham (2019) between the two or more individuals highly engaged in the qualitative research.

This conduct of qualitative research therefore necessitates proximity between the participant and the researcher. But given the social distancing restrictions posed by the COVID-19 pandemic, the collection methods for qualitative data is hampered, and that the accessibility of data source becomes challenging. According to Vindrola-Padros et al. (2010), compared with other research designs, the conduct of qualitative research in the context of infectious epidemics like the COVID-19 pandemic remains to lag in terms of delivery, credibility, and timeliness of findings. This is primarily due to its very nature of high engagement between the participants and the researcher. When social distancing protocols and the threat of coronavirus continues to abide, qualitative researchers have no other choice but to explore other means to collect data while diligently upholding the reliability and credibility of data and academic integrity.

Traditionally, common data collection techniques for qualitative research include direct observation and face-to-face interviews (individual or groups). These are considered the golden standards in qualitative research (Sy et al, 2020). Direct observation facilitates an ethnographic and case study type of research wherein a qualitative researcher involves himself in the day-to-day activities of the participants. Face-to-face interview on the other hand enables the researcher to instantly acquire data from the participants in the exchange of conversation following the semi-structured questionnaire. These two methodologies are not feasible during the COVID-19 pandemic. Hence, adaptive versions of collecting qualitative data need to be employed. 


\subsection{Using diaries and reflections of participants to replace the typical direct observation}

Since direct observation is not possible, the qualitative researcher may just provide reflection guides to the participants so they would be able to generate meaningful and relevant entries into the diary. The diary shall contain the daily reflections or activities undertaken by the participant. In turn, the diary shall become the basis of the qualitative researcher in extracting meaningful constructs and themes. According to Lupton (2020), diaries or reflections from participants in the field can provide a first-hand insight about the experiences of the participant. Further, diary data can be free-flow writing or structured writing in response to specific questions or prompts. diary data can be freeflowing writing or structures. Morell-Scott (2018) expounded that the use of diaries for qualitative research can allow for a concurrent comprehension into the lived experience of the participant completing the diary. During the COVID-19 pandemic, utilizing diaries to replace the direct observation might be the most appropriate for phenomenological or ethnographic researches.

\subsection{Using a telephone or mobile phone to conduct interviews}

When mobility is regulated and face-to-face interviews cannot be conducted to collect qualitative data, qualitative researchers may opt to conduct the interview through a telephone call. In this way, the researcher directly throws the question or scenario to the participant, and the participant directly in-depth answers to the question. There is however a need for the researcher to capture quickly the responses or the answers by writing or jutting down the responses in the field notes. The audio recording may also be considered. According to Drabble (2015), strategies for success in telephone interviews include cultivating rapport and maintaining connection, demonstrating responsiveness to interviewee content, concerns, and communicating regard for the interviewee and her contribution. Lord (2016) however emphasized that in the conduct of telephone interviews, researchers need to be sensitive to the demographic characteristics of telephone interviewees and the anonymity of telephone interviews may be more conducive for discussing sensitive issues.

\subsection{Using video-conferencing for key informant interviews and focus group discussion}

Face-to-face interviews are highly acclaimed in qualitative research because of their ability to elicit honest views on sensitive topics. Considering this amidst the COVID-19 pandemic, video-conferencing can suffice to collect data on real-time, live, and face-toface yet remote. Video conferencing software allows two or more people in different locations to communicate using audio and video imaging in real-time (Gough and Rosenfeld, 2006). In the research of Faherty (2019), he was able to conduct focus group discussion which lasted 90 minutes with school and stakeholders from across the United States. This was a social distancing practice aimed to reduce influenza transmission during a pandemic. At present, other remote and video-conferencing technologies that can replace the actual face-to-face interview may include Skype, Microsoft Teams or 
Zoom, Google Hangout Meet, Collab, Zoho Meeting, GoToMeeting, Cisco WebEx, Highfive Meeting, Eyeson (Gray et al, 2020) or in the form of a webinar. In the analysis of some researchers, it was found out that compared to the traditional face-to-face interview, online video conferencing interviews, online participants were more open and expressive and that rapport between and among participants was built quicker (Deakin and Wakefield, 2013). While it is true that the proliferation of new video conferencing tools offers unique data generation opportunities for qualitative researchers (Gray et al, 2020), it should also be noted that it is more cost-efficient and easy to organize. This also highlights why videoconferencing attracted the utmost attention from qualitative researchers because of its practicality during this time of the pandemic.

\section{Conclusion}

Pursuing research at the time of the COVID-19 pandemic is challenging especially in terms of data collection. Moving away from the traditional methods of data collection had become superfluous to adapt to the restrictions and quarantine protocols implemented by local governments around the world. While it is true that research should not be shuttered amidst the pandemic, the health and safety of the researchers in the field should also be promoted and protected against the coronavirus. Hence, adaptation in the data collection for qualitative and quantitative researches should be reinforced to sustain reliability, honesty, and academic integrity.

\section{About the Author}

Dr. Moises C. Torrentira is an Associate Professor of the University of Southeastern Philippines. He holds PhD in Development Administration, Master in Business Administration, Master in Public Administration, Master in Environmental Planning, BS in Public Administration, and AB in Philosophy. He is a licensed Environmental Planner and licensed Professional Teacher.

\section{References}

Abd Halim, M., Foozy, C. F. M., Rahmi, I., \& Mustapha, A. (2018). A review of the live survey application: SurveyMonkey and SurveyGizmo. JOIV: International Journal on Informatics Visualization, 2(4-2), 309-312.

American Psychological Association (APA) (2020). Conducting research during the COVID-19 pandemic: Advice from psychological researchers on protecting participants, animals, and research plans. American Psychological Association. https://www.apa.org/news/apa/2020/03/conducting-research-covid$\underline{19}$ 
Barnes, L. A. J., Barclay, L., McCaffery, K., Rolfe, M., \& Aslani, P. (2020). Using Facebook to recruit to a national online survey investigating complementary medicine product use in pregnancy and lactation; a case study of method. Research in Social and Administrative Pharmacy.

Bentley, F. R., Daskalova, N., \& White, B. (2017, May). Comparing the reliability of Amazon Mechanical Turk and Survey Monkey to traditional market research surveys. In Proceedings of the 2017 CHI Conference Extended Abstracts on Human Factors in Computing Systems (pp. 1092-1099).

Boas, T. C., Christenson, D. P., \& Glick, D. M. (2020). Recruiting large online samples in the United States and India: Facebook, Mechanical Turk, and qualtrics. Political Science Research and Methods, 8(2), 232-250.

Deakin, H., \& Wakefield, K. (2013). Skype interviewing: Reflections of two Ph.D. researchers. Qualitative Research, 14(5), 603-616. DOI:10.1177/1468794113488126

Dodds, S., \& Hess, A. C. (2020). Adapting research methodology during COVID-19: lessons for transformative service research. Journal of Service Management.

Faherty, L. J., Schwartz, H. L., Ahmed, F., Zheteyeva, Y., Uzicanin, A., \& Uscher-Pines, L. (2019). School and preparedness officials' perspectives on social distancing practices to reduce influenza transmission during a pandemic: Considerations to guide future work. Preventive medicine reports, 14, 100871.

Farooq, M. B., \& De Villiers, C. (2017). Telephonic qualitative research interviews: When to consider them and how to do them. Meditari Accountancy Research.

Flick, U. (2018). An introduction to qualitative research. Sage Publications Limited.

Glazier, R. A., \& Topping, M. P (2012). Using Social Media to Advance Community-based Research. Retrieved from rebeccaglazier.net.

Goertzen, M. J. (2017). Introduction to quantitative research and data. Library Technology Reports, 53(4), 12-18.

Gough, M., \& Rosenfeld, J. (2006). Video conferencing over IP: Configure, secure, and troubleshoot. Rockland, MA: Syngress.

Hennink, M., Hutter, I., \& Bailey, A. (2020). Qualitative research methods. SAGE Publications Limited.

Kimball, S. H. (2019). Survey data collection; online panel efficacy. A comparative study of Amazon MTurk and Research Now SSI/Survey Monkey/Opinion Access. Journal of Business Diversity, 19(2).

Le Blanc, L. (2020). A brief historical review of the great pandemic of 1918: The Spanish flu. Eubios Journal of Asian and International Bioethics, 30 (3), 81-83.

Lord, R., Bolton, N., Fleming, S., \& Anderson, M. (2016). Researching a segmented market: Reflections on telephone interviewing. Management Research Review.

Lupton, D.(ed.) (2020). Doing fieldwork in a pandemic (Crowd-sourced document). https://docs.google.com/document/d/1clGjGABB2h2qbduTgfqribHm og9B6P0NvMgVuiHZCl8/edit?ts=5e88ae0a\#

Morrell-Scott, N. E. (2018). Using diaries to collect data in phenomenological research. Nurse researcher, 25(4), 26-29. 
Padala, P. R., Jendro, A. M., \& Padala, K. P. (2020). Conducting clinical research during the COVID-19 Pandemic: Investigator and participant perspectives. JMIR Public Health and Surveillance, 6(2), e18887.

Ramsbotham, O. (2019). Hans-Georg Gadamer's Truth and Method Revisited: On the Very Idea of a Fusion of Horizons in Intense, Asymmetric, and Intractable Conflicts. Journal of Dialogue Studies, 73.

Remuzzi, A., and Remuzzi, G. (2020). COVID-19 and Italy: what next? The Lancet. https://doi.org/10.1016/S0140-6736(20)30627-9.

Sukamolson, S. (2007). Fundamentals of quantitative research. Language Institute Chulalongkorn University, 1, 2-3.

Sy, M., O'Leary, N., Nagraj, S., El-Awaisi, A., O'Carroll, V., \& Xyrichis, A. (2020). Doing interprofessional research in the COVID-19 era: a discussion paper. Journal of Interprofessional Care, 1-7.

Torrentira, M. (2019). Dimensions of sustainable research collaborations in Philippine Universities, Journal of Public Administration and Governance. Vol. 9 No. 2, pp. 17-29, DOI: http://dx.doi.org/10.5296/jpag.v9i2.14683.

Townsend, E., Nielsen, E., Allister, R., \& Cassidy, S. A. (2020). Key ethical questions for research during the COVID-19 pandemic. The Lancet Psychiatry, 7(5), 381-383.

Vaske, J. J. (2019). Survey research and analysis. Sagamore-Venture. 1807 North Federal Drive, Urbana, IL 61801.

Vernon, M. M., Moore, N., Mazzoli, A., \& De Leo, G. (2018). Respiratory therapy faculty perspectives on interprofessional education: Findings from a cross-sectional online survey. Journal of Interprofessional Care, 32(2), 235238. https://doi.org/10.1080/13561820.2017.1389865

Vindrola-Padros, C., Chisnall, G., Cooper, S., Dowrick, A., Djellouli, N., Symmons, S. M., ... \& Johnson, G. A. (2020). Carrying Out Rapid Qualitative Research During a Pandemic: Emerging Lessons From COVID-19. Qualitative Health Research, 1049732320951526.

WHO (2020). Corononavirus disease (COVID-19). Retrieved from https://www.who.int/emergencies/diseases/novel-coronavirus-

2019?gclid=EAIaIQobCh $\quad$ MI2Ofl3v $\quad$ HY6w


Moises C. Torrentira, Jr.

ONLINE DATA COLLECTION AS ADAPTATION IN CONDUCTING QUANTITATIVE AND QUALITATIVE RESEARCH DURING THE COVID-19 PANDEMIC

Creative Commons licensing terms

Author(s) will retain the copyright of their published articles agreeing that a Creative Commons Attribution 4.0 International License (CC BY 4.0) terms will be applied to their work. Under the terms of this license, no permission is required from the author(s) or publisher for members of the community to copy, distribute, transmit or adapt the article content, providing a proper, prominent and unambiguous attribution to the authors in a manner that makes clear that the materials are being reused under permission of a Creative Commons License. Views, opinions and conclusions expressed in this research article are views, opinions and conclusions of the author(s). Open Access Publishing Group and European Journal of Education Studies shall not be responsible or answerable for any loss, damage or liability caused in relation to/arising out of conflicts of interest, copyright violations and inappropriate or inaccurate use of any kind content related or integrated into the research work. All the published works are meeting the Open Access Publishing requirements and can be freely accessed, shared, modified, distributed and used in educational, commercial and non-commercial purposes under a Creative Commons Attribution 4.0 International License (CC BY 4.0). 